\title{
Overexpression of IFIT2 inhibits the proliferation of chronic myeloid leukemia cells by regulating the BCR-ABL/AKT/mTOR pathway
}

\author{
ZHANGLIN ZHANG ${ }^{1,2^{*}}$, NA LI $^{3 *}$, SHUYUAN LIU ${ }^{2}$, MEI JIANG ${ }^{2}$, JINGHUA WAN $^{2}$, \\ YONGLU ZHANG ${ }^{2}$, LAGEN WAN ${ }^{2}$, CAIFENG XIE $^{4}$ and AIPING LE ${ }^{1}$ \\ Departments of ${ }^{1}$ Blood Transfusion, ${ }^{2}$ Clinal Laboratory and ${ }^{3}$ Stomatology, \\ The First Affiliated Hospital of Nanchang University, Nanchang, Jiangxi 330006; \\ ${ }^{4}$ School of Basic Medical Sciences, Nanchang University, Nanchang, Jiangxi 330031, P.R. China
}

Received August 6, 2019; Accepted December 6, 2019

DOI: $10.3892 / \mathrm{ijmm} .2020 .4500$

\begin{abstract}
Chronic myeloid leukemia (CML) is a myeloproliferative disorder that accounts for $\sim 10 \%$ of all newly diagnosed leukemia cases. Early diagnosis is essential for long-term beneficial outcomes. The present study observed that interferon-induced protein with tetratricopeptde repeats 2 (IFIT2) expression levels were reduced in bone marrow samples from CML patients compared with control samples using RNA sequencing and reverse transcription-PCR. IFIT2 expression levels were restored in patients treated with tyrosine kinase inhibitors. To investigate the effect of IFIT2 on CML patients, a stable IFIT2 expressing K562 cell line was established. It was demonstrated that IFIT2 overexpression in K562 cells inhibits cell proliferation and arrests the cell cycle at the G1 phase. In addition, it was demonstrated by western blotting that IFIT2 inhibits the BCR-ABL oncoprotein and regulates its downstream AKT/mTOR signaling pathway. IFIT2 could induce cell cycle arrest-associated gene $\mathrm{p} 27^{\mathrm{kipl}}$ by degrading cullin1-mediated E3 ligases. In summary, the present study demonstrated that IFIT2 was efficacious in inhibiting CML and is a potential therapeutic target.
\end{abstract}

Correspondence to: Dr Aiping Le, Department of Blood Transfusion, The First Affiliated Hospital of Nanchang University, 17 Yongwai Zhen Street, Nanchang, Jiangxi 330006, P.R. China E-mail: leaiping@126.com

Dr Caifeng Xie, School of Basic Medical Sciences, Nanchang University, 1299 Xuefu Road, Honggu Tan, Nanchang, Jiangxi 330031, P.R. China

E-mail: xiecaifeng@ncu.edu.cn

${ }^{*}$ Contributed equally

Key words: interferon-induced protein with tetratricopeptde repeats 2, chronic myeloid leukemia, BCR-ABL, K562 cells

\section{Introduction}

Chronic myeloid leukemia (CML) is a myeloproliferative disease that is characterized by an increase in immature myeloid cells in the bone marrow and peripheral blood (1). CML is associated with a reciprocal translocation $\mathrm{t}(9 ; 22)$ (q34;q11), which results in the generation of a BCR/ABL fusion gene (2). The BCR-ABL fusion gene encodes a tyrosine kinase oncoprotein that induces cell proliferation and leukemogenesis by activating the RAS, PI3K/AKT, ERK, MYC and JAK/STAT signaling pathways (3). The tyrosine kinase inhibitor (TKI) imatinib (IM) has been demonstrated to inhibit the kinase activity of BCR-ABL and has been used as a first-line treatment strategy for CML (4). However, $35 \%$ of CML patients exhibit disease progression, relapse and/or intolerance to IM (5). The majority of primary and secondary resistance mechanisms include the amplification of BCR-ABL and point mutations within the ABL kinase domain (6-8). Second and third generation TKIs, such as dasatinib and ponatinib, are able to overcome IM resistance in some patients with T315I mutations (9-11). However, these TKIs have severe side effects, such as cardiovascular thrombotic events, and have therefore been removed from the market. There is an urgent need to develop novel therapeutic strategies with high efficacy and low toxicity for the treatment of patients with CML.

Interferon $\alpha$ (IFN $\alpha$ ) has been the treatment choice for certain patients with CML (12). Therefore, TKIs, IFN $\alpha$ or a combination strategy may emerge as a treatment option for CML (13). Interferon-induced protein with tetratricopeptide repeats 2 (IFIT2), also known as ISG54, is a member of the IFN-stimulated genes. IFIT2 was originally identified as a direct response target to type I IFN, and it has been reported to serve a crucial role in host antiviral defense in the innate immune response (14). IFIT2 is considered a tumor suppressor in several tumor types, such as oral squamous cell carcinoma, breast cancer and leukemia, as it has been identified to inhibit cancer cell growth and migration, and promote cell apoptosis. In addition, IFIT2 has been demonstrated to be associated with clinical parameters and therapeutic outcomes $(15,16)$. Our previous study (16) revealed that IFIT2 overexpression 
could enhance curcumin-induced apoptosis in the CML cell line K562. However, whether IFIT2 expression inhibits CML progression remains to be determined.

Using RNA sequencing arrays, the present observed that specific interferon signaling pathway-associated genes, including IFIT2, had reduced expression levels in CML patients compared with healthy controls. Therefore, the current study investigated the role of IFIT2 in CML.

\section{Materials and methods}

CML and normal bone marrow cells. Bone marrow cells that were isolated from 26 patients with primary CML were obtained from the Department of Hematology, First Affiliated Hospital of Nanchang University (Nanchang, China) between January 2016 and December 2017. All patients were diagnosed with CML during the chronic phase using the Morphology, Immunology, Cytogenetics and Molecular (MICM) criteria of the World Health Organization classification of myeloid neoplasms (17). Patients were identified as harboring BCR-ABL transcripts using karyotype analysis, fluorescence in situ hybridization and/or reverse transcription (RT)-PCR. All patients included in the study were administered IM $(400 \mathrm{mg}$ p.o. q.d.) as a front-line treatment, and the copies of BCR/ABL were monitored by PCR every 3 months. Additionally, 16 bone marrow samples as controls were collected from patients with normal bone marrow, diagnosed by the MICM criteria, at the Department of Hematology, First Affiliated Hospital of Nanchang University between January 2016 and December 2017. No significant differences were identified between the sex and age of the two groups (Table I). The present study was approved by the Medical Ethics Committee of the First Affiliated Hospital of Nanchang University (Nanchang, China). All the patients provided written informed consent.

Library construction and RNA sequencing. Total RNA from the bone marrow of patients was extracted using TRIzol reagent (Thermo Fisher Scientific, Inc.), according to the manufacturer's protocol. RNA integrity was determined using the Agilent Bioanalyzer 2100 (Agilent Technologies, Inc.). RNA-sequencing library construction was performed using the standard TruSeq RNA sample preparation v2 protocol (Illumina, Inc.). Libraries were then sequenced using the Illumina HiSeq2500 platform at Shanghai Biotechnology Corporation (Shanghai, China). Sequencing and real-time data analyses were performed using the StringTie software (version 1.3.0) provided by Illumina.

Cell culture and reagents. The human CML cell line K562 was cultured in RPMI-1640 media (Gibco; Thermo Fisher Scientific, Inc.) supplemented with $10 \%$ fetal bovine serum (Hyclone; GE Healthcare Life Sciences). The 293T cell line was cultured in DMEM (Gibco; Thermo Fisher Scientific, Inc.) supplemented with $10 \%$ fetal bovine serum. Cells were cultured in a $37^{\circ} \mathrm{C}$ incubator with $5 \% \mathrm{CO}_{2}$. Doxycycline (DOX) and puromycin were purchased from Sigma-Aldrich; Merck KGaA and Invitrogen; Thermo Fisher Scientific, Inc., respectively. RT reagent (cat. no. KR103), Super-Real qPCR PreMix (SYBR-Green) reagent kit (cat. no. FP205) and PCR 2xMix reagent (cat. no. KT201) were purchased from Tiangen Biotech
Co.,Ltd. pLVX-tetone-puro lentiviral expression vector and the lentiviral packaging plasmids were purchased from Clontech Laboratories, Inc. Antibodies against p27 (cat. no. sc-53906; 1:500) and IFIT2 (cat. no. sc-390724; 1:500), goat anti-mouse IgG-HRP (cat. no. sc-2005; 1:10,000) and goat anti-rabbit IgG-HRP (cat. no. SC-2004; 1:10,000) were purchased from Santa Cruz Biotechnology. Anti-cullin-1 (cat. no. 71-8700; 1:1,000) was purchased from Invitrogen; Thermo Fisher Scientific, Inc. Anti-CSN3 (cat. no. GTX33109; 1:5,000) and anti-CSN5 (cat. no. GTX70203; 1:1,000) were purchased from GeneTex, Inc. Antibodies against mTOR (cat. no. 2972; 1:1,000), phosphorylated (p)-mTOR (cat. no. 2971; 1:1,000), AKT (cat. no. 9272; 1:1,000) and p-AKT (cat. no. 9271; 1:1,000) were purchased from Cell Signaling Technology, Inc. Antibodies against hemagglutinin (HA) (cat. no. AE008; 1:5,000), ABL proto-oncogene 1, non-receptor tyrosine kinase (ABL1; cat. no. A0282; 1:500), c-Jun (cat. no. A0246; 1:500), p-c-Jun-ser63 (cat. no. AP0048; 1:500) and $\beta$-actin (cat. no. AC038; 1:50,000) were purchased from Abclonal Technology, Inc.

Plasmid construction, transfection and lentiviral transduction. The IFIT2 open reading frame (ORF) was cloned in-frame to the $\mathrm{HA}$ tag at the $\mathrm{N}$-terminal to generate the pEGFP-C1-IFIT2 plasmid. The vector pEGFP-C1 was from Clontech Laboratories, Inc. The IFIT2 ORF was amplified using PCR with PCR 2xMix reagent and the following primers: Sense, 5'-AGTCCAATTGCCACCATGGTGTAC CCATACGACGTCCCAGACTACGCTAGTGAGAACAAT AAC-3' and antisense, 5'-TATGGATCCTCAGCAGTAGC CTA-3'. The HA-IFIT2 complementary DNA (cDNA) was then inserted into the lentiviral vector pLVX-tetone-puro, and then transfected into $293 \mathrm{~T}$ cells together with the packing plasmids pSPAX2 and pMD2G at a ratio of 5:3:2 using EndoFectin ${ }^{\mathrm{TM}}$ (GeneCopoeia, Inc.) based on the manufacturer's protocol. A total of $48 \mathrm{~h}$ post-transfection, virus was harvested and used to infect K562 cells. Stable clones expressing HA-IFIT2 (IFIT2-K562) and negative control (K562-TETONE) were then selected using puromycin. Stable cell clones transduced with the empty vector were used as the controls. The expression of HA-IFIT2 was measured after treating with $2 \mu \mathrm{g} / \mathrm{ml}$ Doxycycline (cat. no. 24390-14; MedChemExpress).

Cell cycle analysis. Cell cycle analysis was performed as described previously (18). Briefly, $1 \times 10^{6}$ cells stably expressing IFIT2 or control cells were harvested and then stained using the COULTER DNA Prep Reagents kit (Beckman Coulter, Inc.), according to the manufacturer's protocol. Stained cells were analyzed using a flow cytometer and cell cycle analysis was performed using MODFIT LT 4.1 software (Verity Software House).

Immunofluorescence staining. Stable cells were transferred onto slides using cytospin and fixed with $4 \%$ paraformaldehyde for $15 \mathrm{~min}$ at room temperature. Following permeabilization with $0.5 \%$ Triton X-100 in PBS for 5 min at room temperature, cells were incubated with primary antibody anti-IFIT2 (1:200) for $2 \mathrm{~h}$ at $37^{\circ} \mathrm{C}$. Slides were then washed three times with PBST and incubated with FITC-conjugated anti-mouse IgG (1:100; 
cat. no. AB_2769475; Abclonal Technology, Inc.) for $1 \mathrm{~h}$ at room temperature. Nuclear staining was performed using 4,6-diamidino-2-phenylindole for $5 \mathrm{~min}$ at room temperature. Slides were then washed with PBST and fluorescent images were observed using a fluorescence microscope (Olympus Corporation).

RT-quantitative PCR ( $q P C R)$. Total RNA was extracted using TRIzol (cat. no. 15596026; Invitrogen; Thermo Fisher Scientific, Inc.) and reverse transcribed to cDNA at $42^{\circ} \mathrm{C}$ for $1 \mathrm{~h}$. Gene expression levels for IFIT2 were measured by qPCR with Super-Real qPCR PreMix reagent at the following conditions: $95^{\circ} \mathrm{C}$ for $5 \mathrm{~min}$, and $95^{\circ} \mathrm{C}$ for $5 \mathrm{sec}$ and $60^{\circ} \mathrm{C}$ for $30 \mathrm{sec}$ for 40 cycles. Normalization was performed using $\beta$-actin expression levels. Primers used for qPCR were as follows: IFIT2 forward, 5'-CTGCAACCATGAGTGAGAAC-3' and reverse, 5'-CAG-GTGACCAGACTTCTGAT-3'; and $\beta$-actin forward, 5'-CATGTACGTTGCTATCCAGGC-3' and reverse, 5'-CTC CTTAATGTCACGCACGAT-3'. Relative expression levels were calculated using the comparative $2^{-\Delta \Delta \mathrm{Cq}}$ method (19).

Western blot analysis. Total proteins were extracted using lysis buffer [1\% Triton X-100, $50 \mathrm{mM}$ Tris (pH 8.0), $150 \mathrm{mM} \mathrm{NaCl}$, $1 \mathrm{mM}$ PMSF, $1 \mathrm{mM} \mathrm{Na} 3 \mathrm{VO} 4$ and protease inhibitor cocktail). Protein concentrations were quantified using the Bio-Rad Protein assay kit II (cat. no. 5000002; Bio-Rad Laboratories, Inc.). Total protein (20 $\mu \mathrm{g} /$ lane) was separated using $10 \%$ SDS-PAGE gel, and then electro-transferred to a nitrocellulose membrane. Membranes were blocked for $1 \mathrm{~h}$ at room temperature in Tris-buffered saline- $0.05 \%$ Tween-20 (TBST) containing $5 \%$ non-fat dry milk. Subsequently, the membranes were incubated with primary antibodies overnight at $4^{\circ} \mathrm{C}$ The membranes were washed three times with TBS for $10 \mathrm{~min}$, and then incubated with peroxidase-conjugated secondary antibody at room temperature for $1 \mathrm{~h}$. Following three additional 10 min washes with TBST, target proteins were detected using enhanced chemiluminescence detection reagent (EMD Millipore) and imaged using the Bio-Rad ChemiDoc XRS+ chemiluminescence imaging system (Bio-Rad laboratories, Inc.).

Statistical analysis. Student's t-test was used to compare the expression of IFIT2 in patients and control groups. One-way ANOVA was used to analyze the expression of IFIT2 in the bone marrow of the patients following treatment with TKI for different durations. Two-way ANOVA was used to analyze the differences in proliferation and cell cycle of the cells following stable expression of IFIT2 and treatment with DOX. Following ANOVA, Bonferroni's post hoc test was used to determine significant differences. All data are presented as mean \pm standard deviation. $\mathrm{P}<0.05$ was considered to indicate a statistically significant difference. Statistical analyses were performed using GraphPad Prism 6 (GraphPad Software, Inc.).

\section{Results}

IFIT2 expression levels are downregulated in CML patients and upregulated following TKI administration. To determine the mechanism of leukemogenesis in CML, the present
Table I. Age and sex distribution of controls and patients with CML.

\begin{tabular}{lccc}
\hline & Control $(\mathrm{n}=16)$ & CML $(\mathrm{n}=26)$ & P-value \\
\hline Sex & & & 0.9998 \\
Male & 7 & 11 & \\
Female & 9 & 15 & \\
Age, years & $19-70$ & $24-71$ & 0.3861 \\
\hline CML, chronic myeloid leukemia. & & \\
\hline
\end{tabular}

study performed RNA-sequencing analysis of bone marrows obtained from three primary CML patients and health donors. Gene expression analysis demonstrated that a number of IFN signal pathway-related genes were differentially expressed in CML compared healthy controls. This included several well-known IFN-induced genes, such as IFIT3, IFIT2, IRF1, IFITM3, OAS2 and OAS1 (Fig. 1A). Among them, IFIT2 mRNA levels were markedly downregulated in CML patients. To validate these results, IFIT2 mRNA expression levels were measured in bone marrow cells from 26 patients with primary CML and 16 healthy controls by RT-qPCR. As presented in Fig. 1B, IFIT2 expression levels were significantly lower in CML patients compared with controls. In addition, IFIT2 expression levels were measured in CML patients treated with TKI. In 6 patients with CML who had been treated with TKI for 6 months, IFIT2 expression levels were higher compared with newly diagnosed patients (Fig. 1C). These results indicated that IFIT2 levels were decreased in CML patients and upregulated in patients treated with TKIs.

Stable K562 cell lines expressing IFIT2. To functionally investigate the role of IFIT2 in CML, the present study established a stable IFIT2-K562 cell line that overexpressed IFIT2 following DOX exposure. HA-IFIT2 ORF was cloned into pLVX-Tetone-Puro vector with the Tet-On system, which induced gene expression in the presence of DOX (Fig. 2A). Recombinant lentiviruses were produced using 293T cells that were subsequently used to infect K562 cells. Following puromycin selection, western blot and immunofluorescence assays were performed to confirm IFIT2 expression in the selected stable cell lines. As presented in Fig. 2B, the HA-IFIT2 protein was detected using either IFIT2 or HA antibodies in IFIT2-K562 cell lines after $24 \mathrm{~h}$ of DOX exposure $(2 \mu \mathrm{g} / \mathrm{ml})$. IFIT2 was not detected in the vector control cell lines K562-TETONE (Fig. 2B). Immunofluorescence staining using IFIT2 antibody also demonstrated increased IFIT2 protein expression in the cytoplasm of IFIT2-K562 cells following DOX exposure (Fig. 2C).

IFIT2 inhibits cell proliferation by inducing G1 phase arrest in K562 cells. The effect of IFIT2 overexpression on the proliferation of K562 cells was then measured. The proliferation rates of the K562-TETONE and K562-IFIT2 cell lines were similar (Fig. 3A). Following exposure to $2 \mu \mathrm{g} / \mathrm{ml}$ DOX, cell proliferation in K562-TETONE cells was 
A

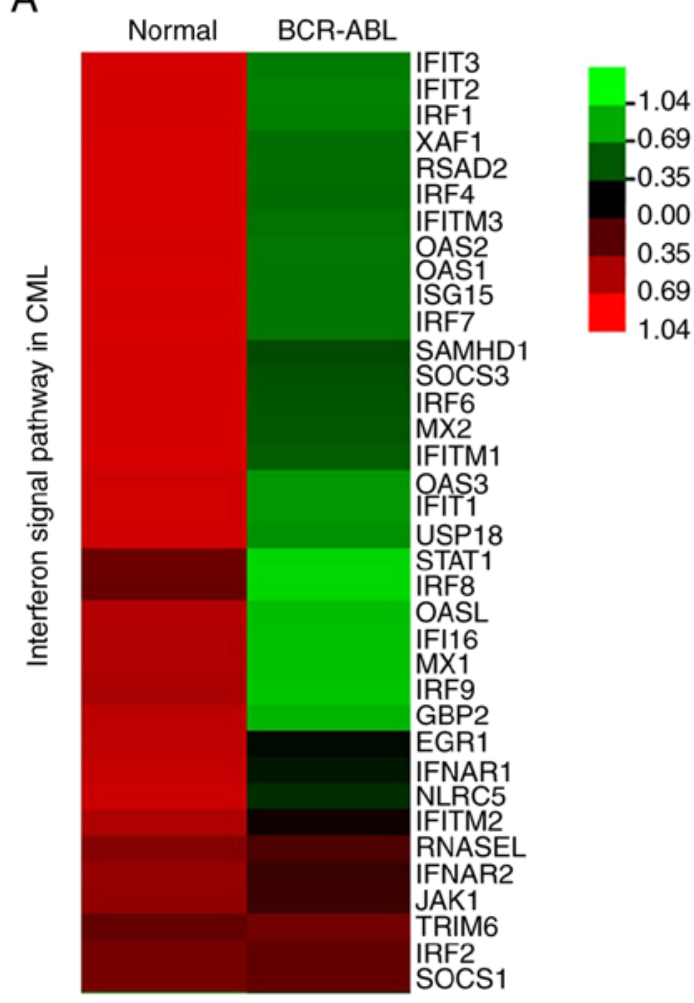

$\mathrm{B}$

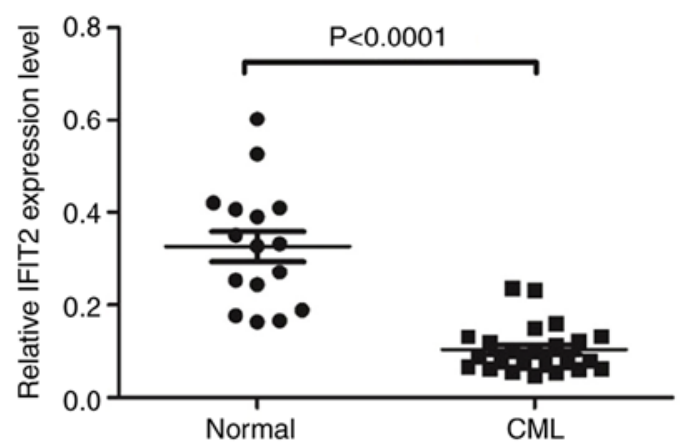

C

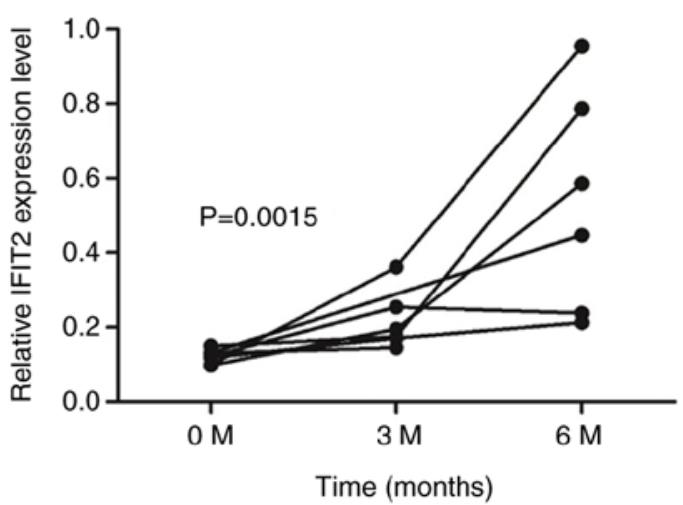

Figure 1. IFIT2 expression levels are reduced in CML patients and upregulated following TKI administration. (A) A gene expression heatmap of the interferon signaling pathway generated using bone marrow samples obtained from patients with CML and control samples. (B) IFIT2 mRNA expression levels in CML patients and controls determined using reverse transcription-quantitative PCR. (C) IFIT2 expression levels in CML patients following TKI administration. IFIT2, interferon-induced protein with tetratricopeptde repeats 2; CML, chronic myeloid leukemia; TKI, tyrosine kinase inhibitor.

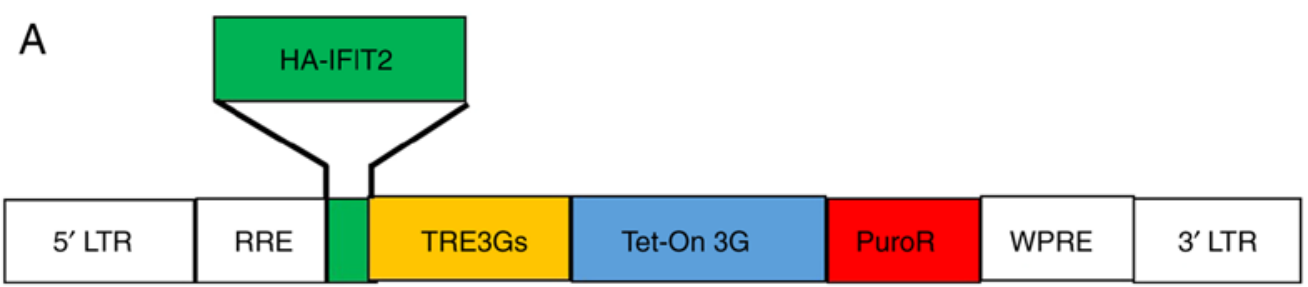

pLVX-Tetone-Puro vector (clontech)
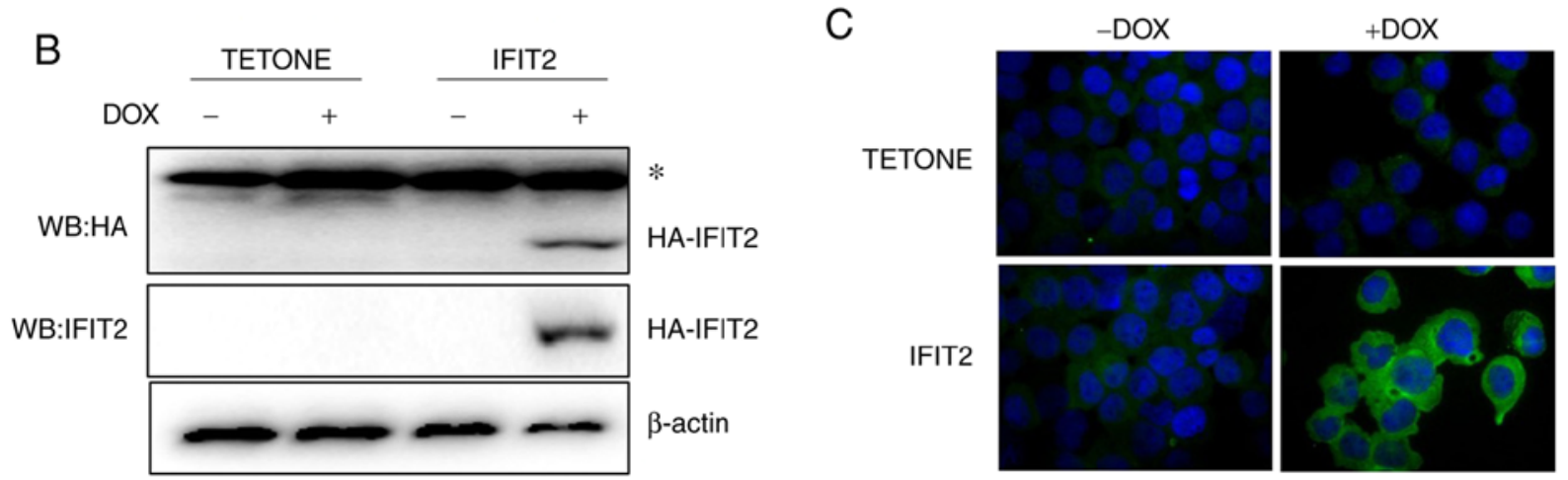

Figure 2. Generation of stably transfected IFIT2-expressing K562 cell lines. (A) pLVX-Tetone-HA-IFIT2 expression plasmid. (B) IFIT2 expression was measured using western blotting. The asterisk indicates a non-specific band. (C) IFIT2 expression was localized and analyzed using immunofluorescence staining (magnification, x100). IFIT2, interferon-induced protein with tetratricopeptde repeats 2; DOX, doxycycline; HA, hemagglutinin.

only slightly reduced due to DOX cytotoxicity; however, proliferation of K562-IFIT2 cells was significantly reduced at 48 and $72 \mathrm{~h}$ compared with K562-TETONE cells treated with DOX $(\mathrm{P}<0.0001)$. Cell cycle analysis demonstrated that IFIT2 overexpression induced growth arrest at the G1 phase in K562 cells. The two cell lines had a similar cell cycle phase 
B
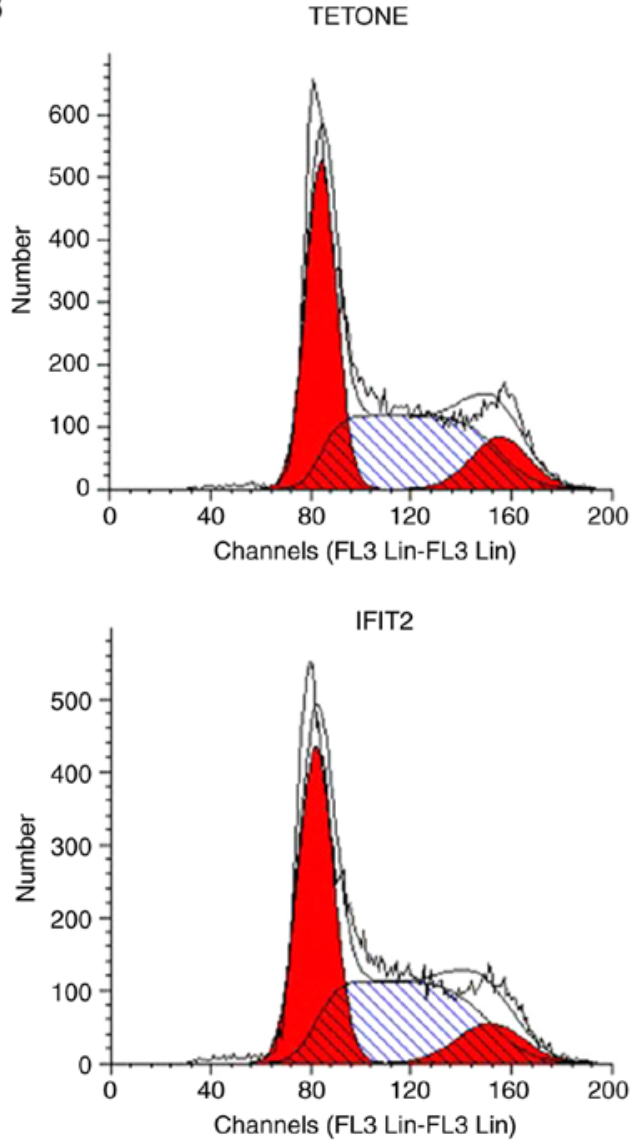

C

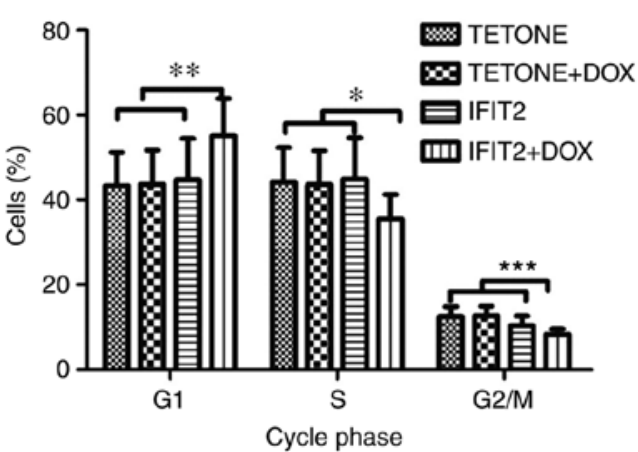

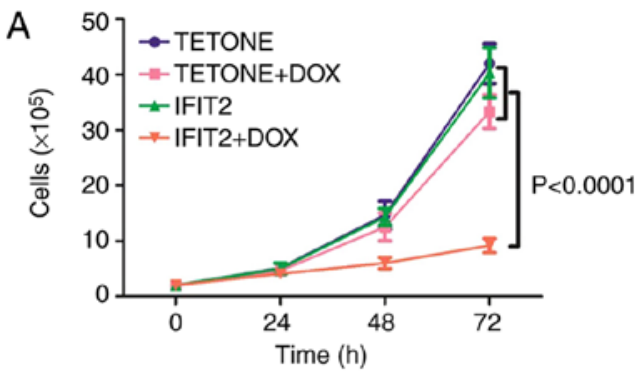

TETONE+DOX

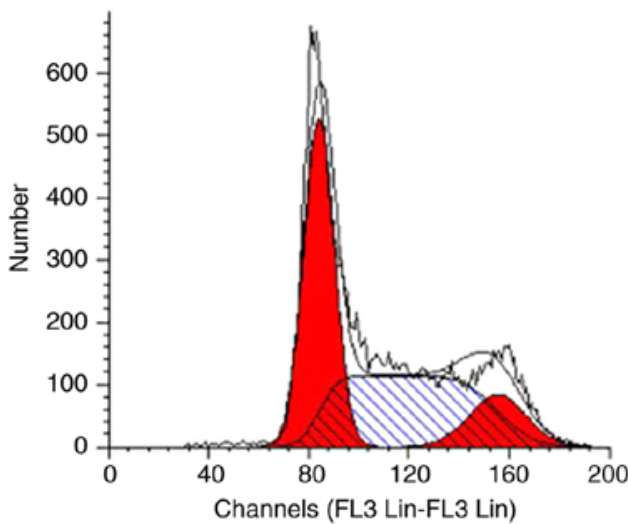

IFIT2+DOX

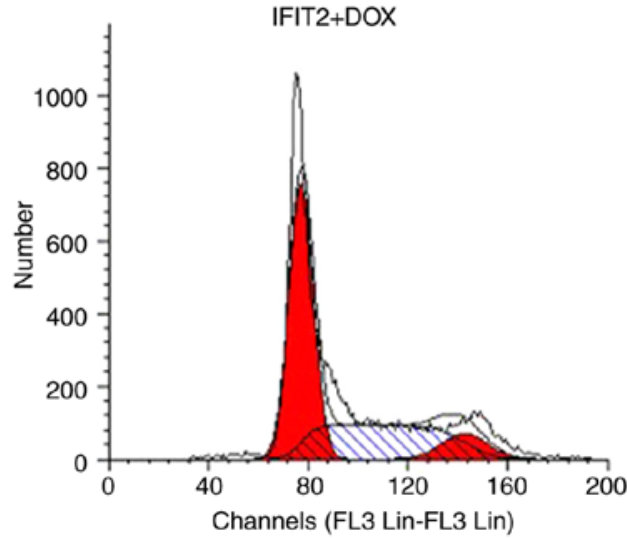

$72 \mathrm{~h}$

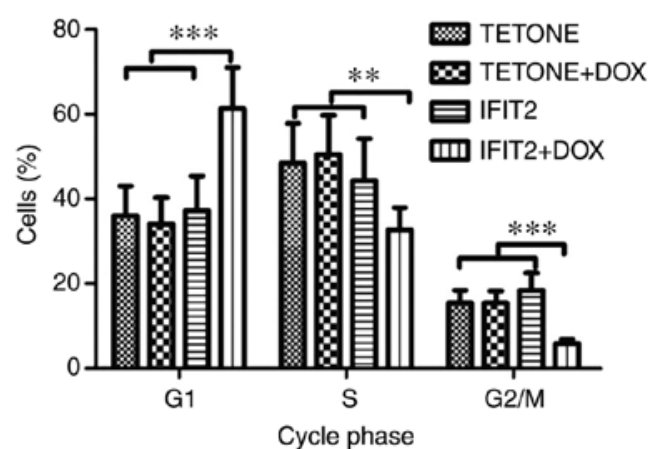

Figure 3. IFIT2 overexpression inhibits K562 cell proliferation and arrests cells at the G1 phase. TETONE-K562 and IFIT2-K562 cells were treated with or without DOX $(5 \mu \mathrm{g} / \mathrm{ml})$ for the indicated times. (A) Growth curves of the stable K562 cell lines at 24, 48 and $72 \mathrm{~h}$. (B) Cell cycle was analyzed using flow cytometry. Percentage of cells in G1, S and G2/M phases of the cell cycle following DOX treatment for (C) 48 and (D) $72 \mathrm{~h} .{ }^{*} \mathrm{P}<0.05,{ }^{* * *} \mathrm{P}<0.01,{ }^{* * * *} \mathrm{P}<0.005$. IFIT2, interferon-induced protein with tetratricopeptde repeats 2; DOX, doxycycline.

distribution in the absence of DOX (Fig. 3B and C). However, treatment of K562-IFIT2 cells with DOX for $48 \mathrm{~h}$ resulted in an obvious accumulation of the cells at G1 transition phase $(\mathrm{P}<0.01)$. Moreover, a significant increase of K562-IFIT2 cells was present in G1 phase following treatment with DOX for $72 \mathrm{~h}(\mathrm{P}<0.001$; Fig. 3D). These results demonstrated that IFIT2 inhibits cell proliferation and arrests cells at the G1 phase in CML cells. 


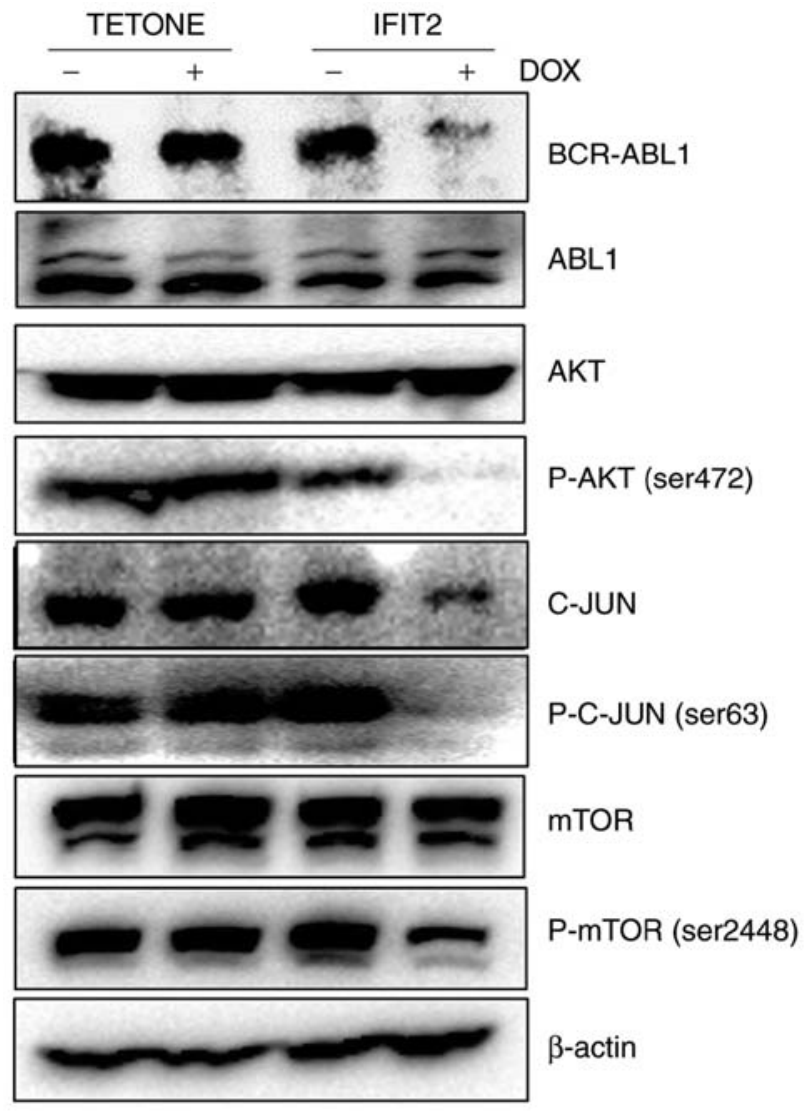

Figure 4. IFIT2 overexpression reduces BCR-ABL expression and inhibits phosphorylation of mTOR and c-Jun. K562-TETONE and K562-IFIT2 cells were treated with or without DOX $(2 \mu \mathrm{g} / \mathrm{ml})$ for $72 \mathrm{~h}$, and then the expression levels of ABL1, BCR-ABL1, AKT, p-AKT (ser472), c-Jun, p-c-Jun (ser63), mTOR and p-mTOR were analyzed by western blotting. $\beta$-actin expression levels were used as the loading controls. IFIT2, interferon-induced protein with tetratricopeptde repeats 2; DOX, doxycycline; p-, phosphorylated; ABL, $\mathrm{ABL}$ proto-oncogene 1, non-receptor tyrosine kinase.

IFIT2 reduces BCR-ABL expression and inhibits phosphorylation of $A K T / m T O R$. The present study then assessed the effect of IFIT2 on the expression of BCR/ABL fusion protein and its downstream targets in K562 cells. As presented in Fig. 4, the two cell lines had similar BCR-ABL expression levels in the absence of DOX treatment. However, BCR-ABL protein expression was markedly decreased in IFIT2-K562 cells following DOX exposure, while ABL1 expression levels were unchanged for both stable cell lines. It has been demonstrated that several signal transduction pathways are activated by BCR/ABL, such as the JAK/STAT/AKT, PI3K/AKT/mTOR and ERK/MAPK/JNK pathways (3). These are critical pathways required for $\mathrm{CML}$ cell proliferation. To determine whether IFIT2 affects any of these signaling pathways, western blot analysis was performed. IFIT2 overexpression markedly reduced the protein levels of c-Jun and p-c-Jun. However, it had no effect on AKT or mTOR protein levels, but reduced the phosphorylation levels of p-AKT and p-mTOR. These results demonstrated that inhibition of cell proliferation induced by IFIT2 in CML cells was associated with reduced expression of $\mathrm{BCR} / \mathrm{ABL}$ and inhibition of the BCR-ABL/AKT/mTOR signaling pathway.

IFIT2 increases p27kipl expression by inhibiting CRL1-E3 ligase. BCR-ABL inhibits $\mathrm{p} 27^{\mathrm{kip} 1}$ via the MAPK and PI3K

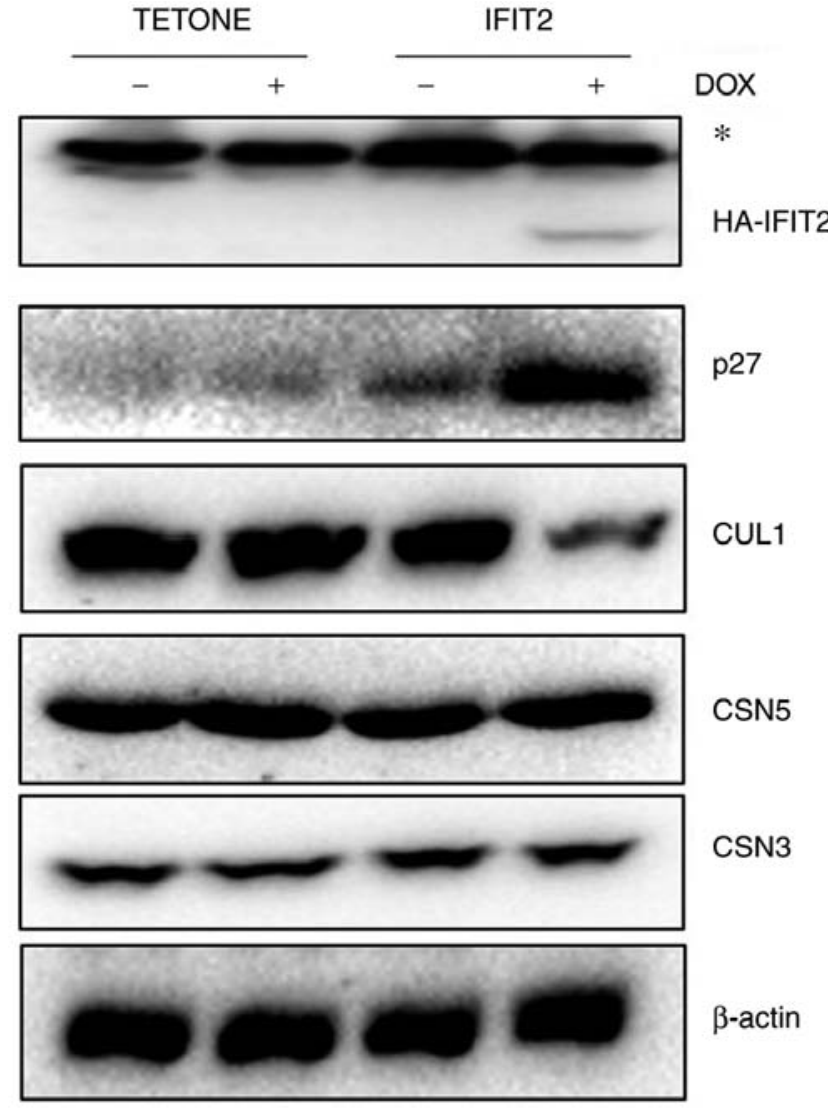

Figure 5. IFIT2 overexpression increases p27 expression levels by inhibiting CRL-E3 ligase. TETONE-K562 and IFIT2-K562 cells were treated with or without DOX $(2 \mu \mathrm{g} / \mathrm{ml})$ for $72 \mathrm{~h}$, and then the expression levels of HA-IFIT2, p27, CUL1, CSN3 and CSN5 were measured using western blotting. $\beta$-actin was used as the loading control. The asterisk indicates a non-specific band. IFIT2, interferon-induced protein with tetratricopeptide repeats 2; DOX, doxycycline; HA, hemagglutinin; CRL, cullin1-RING ligase; CUL1, cullin 1.

signaling pathways and accelerates abnormal cell proliferation leading to leukemogenesis (20). The present study hypothesized that IFIT2 could upregulate $\mathrm{p} 27^{\mathrm{kip} 1}$ protein expression by inhibiting BCR-ABL. Using western blot analysis, it was observed that $\mathrm{p} 27^{\mathrm{kip} 1}$ protein levels were markedly increased in K562-IFIT2 cells following DOX exposure $(2 \mu \mathrm{g} / \mathrm{ml})$. Cell-cycle inhibitor p27 is a known substrate of cullin1-RING ligase (CRL1). CRL1 belongs to a large family of ubiquitin E3 ligases that regulate protein degradation via the ubiquitin proteasome system (21). As presented in Fig. 5, CUL1 levels were markedly decreased in K562-IFIT2 cells following DOX exposure compared with control cells. However, CSN3 and CSN5 levels were similar between the two cell lines, in the presence or absence of DOX. These results demonstrated that IFIT2 promotes the accumulation of $\mathrm{p} 27^{\mathrm{kip} 1}$ by inhibiting BCR-ABL tyrosine kinase activity and degrading cullin1-mediated E3 ligases to suppress CML cell proliferation.

\section{Discussion}

In humans, IFITs consist of four members, IFIT1, IFIT2, IFIT3 and IFIT5. In addition to antiviral activity, IFIT2 has been reported to inhibit tumor cell growth, migration and metastasis in a variety of tumor types (22-24). Our previous 
study demonstrated that curcumin induces cell apoptosis via an IFIT2-dependent signaling pathway in U937 leukemia cells but not in K562 cells. In addition, our previous study demonstrated that upregulation of IFIT2 by exogenous methods or treatment with IFN $\gamma$ in K562 cells increases apoptosis and enhances the anticancer effects of curcumin (25). As the extraction of bone marrow is an invasive method, it was difficult to collect bone marrow specimens from healthy volunteers as controls. In the present study, the expression of IFIT2 in the bone marrow of CML patients was compared with that in normal controls that were diagnosed with iron deficiency anemia and immune thrombocytopenia, and whose bone marrow samples were normal according to the MICM criteria. It was demonstrated that IFIT2 levels are decreased in the bone marrow of CML patients, while patients treated with TKI therapy exhibited increased levels. This suggests that IFIT2 may play an important role in leukemogenesis and could be a potential therapeutic target for CML.

The present study established a CML stable cell line, which was a K562 cell line that stably expressed IFIT2. Using this stable cell line, it was demonstrated that IFIT2 overexpression inhibited cell proliferation and arrested cells at the G1 phase. In addition, it was demonstrated that IFIT2 decreased BCR-ABL expression and inhibited the phosphorylation of AKT, mTOR and c-JUN. Previous studies have demonstrated that constitutive tyrosine kinase activity of BCR-ABL contributes significantly to leukemogenesis in CML. BCR-ABL promotes the survival, proliferation and adhesion of leukemic cells by regulating downstream pathways. Multiple intracellular signal transduction pathways, including the JAK/STAT, ERK/MAPK and $\mathrm{PI} 3 \mathrm{~K} / \mathrm{AKT}$ pathways, have been implicated in this process. Huang et al (26) demonstrated that EPS8 regulates the proliferation, apoptosis and chemo-sensitivity of BCR-ABL positive cells via the BCR-ABL/PI3K/AKT/mTOR pathway. This suggests that IFIT2 may suppress the proliferation of CML cells by regulating the BCR-ABL/AKT/mTOR pathway.

The present study also demonstrated that IFIT2 overexpression induced an accumulation of $\mathrm{p} 27^{\mathrm{kip} 1}$ protein and inhibited CRL1-E3 ligase. $\mathrm{p} 27^{\mathrm{kip} 1}$ is a potent inhibitor of cyclin-dependent kinases that drives G1 to $\mathrm{S}$ phase transition (27). The degradation of $\mathrm{p} 27^{\mathrm{kip} 1}$ protein is mainly regulated through an ubiquitination proteasome dependent pathway associated with cullin 1-mediated E3 ligases (21). The current results demonstrated the involvement of cullin 1-mediated E3 ligases in the upregulation of $\mathrm{p} 27^{\mathrm{kip} 1}$. A previous study has demonstrated that $\mathrm{p} 27^{\mathrm{kip} 1}$ deficiency increases the HSC-containing $\mathrm{Lin}-\mathrm{Sca}-1^{+} \mathrm{c}-\mathrm{Kit}^{+}$cell population and accelerates leukemogenesis in CML mouse models (28). Therefore, increased p27 ${ }^{\mathrm{kipl}}$ levels may inhibit K562 proliferation. Previous studies have reported that inhibition of the nuclear import of $\mathrm{p} 27^{\mathrm{kip} 1}$ by protein kinase B/Akt-mediated phosphorylation is associated with poor prognosis in numerous breast cancer patients $(29,30)$. Tomoda et al (20) demonstrated that BCR-ABL tyrosine kinase facilitates the downregulation of p2 $7^{\text {kipl }}$ by modulating complex formation of Jab1/CSN through the MAPK and PI3K signaling pathways. The present study found that overexpression of IFIT2 in K562 cells decreased c-Jun, p-c-Jun and p-AKT levels. c-Jun, a critical component of the transcription factor AP-1, plays an important role in cell cycle progression, differentiation and cell transformation, and is a downstream target of the PI3K/AKT signaling pathway (31).
AP-1 blockade has been shown to arrest the cell cycle by inducing the expression of p27 in cancer cells $(31,32)$. This suggests that the PI3K/AKT/c-Jun signaling pathway may be involved in the increased expression of $\mathrm{p} 27$.

In conclusion, the present study demonstrated that IFIT2 inhibits CML cell proliferation and arrests the cell cycle at the G1 phase. The underlying mechanism is via regulation of p27 and inhibition of the BCR-ABL/AKT/mTOR signaling pathway. Therapeutic targeting of the signaling pathways that modulate IFIT2 expression may improve clinical outcomes for patients with CML.

\section{Acknowledgements}

Not applicable.

\section{Funding}

This work was supported by the Natural Science Foundation of China (grant. nos. 81760539 and 81760381), Natural Science Foundation of Jiangxi Province (grant. no. 20151BAB205020) and Science and Technology Plan Project of Jiangxi Provincial Health Planning Commission (grant. no. 20171045).

\section{Availability of data and materials}

The datasets generated and/or analyzed during this study are included in this published article.

\section{Authors' contributions}

AL contributed to the study design. ZZ, NL, SL, MJ and JW preformed the in vitro and in vivo experiments. YZ, LW and $\mathrm{CX}$ performed the data analysis. ZZ, CX and AL wrote the manuscript. All authors read and approved the final manuscript.

\section{Ethics approval and consent to participate}

The present study was approved by the Medical Ethics Committee of the First Affiliated Hospital of Nanchang University (Nanchang, China). All patients provided written informed consent.

\section{Patient consent for publication}

Not applicable.

\section{Competing interests}

The authors declare that they have no competing interests.

\section{References}

1. Faderl S, Kantarjian HM and Talpaz M: Chronic myelogenous leukemia: Update on biology and treatment. Oncology (Williston Park) 13: 169-180; discussion 181, 184, 1999.

2. Ben-Neriah Y, Daley GQ, Mes-Masson AM, Witte ON and Baltimore D: The chronic myelogenous leukemia-specific P210 protein is the product of the bcr/abl hybrid gene. Science 233: 212-214, 1986.

3. Ren R: Mechanisms of BCR-ABL in the pathogenesis of chronic myelogenous leukaemia. Nat Rev Cancer 5: 172-183, 2005. 
4. Druker BJ, Guilhot F, O'Brien SG, Gathmann I, Kantarjian H, Gattermann N, Deininger MW, Silver RT, Goldman JM, Stone RM, et al: Five-year follow-up of patients receiving imatinib for chronic myeloid leukemia. N Engl J Med 355: 2408-2417, 2006.

5. Santos FP, Kantarjian H, Quintas-Cardama A and Cortes J: Evolution of therapies for chronic myelogenous leukemia. Cancer J 17: 465-476, 2011.

6. Zabriskie MS, Eide CA, Tantravahi SK, Vellore NA, Estrada J, Nicolini FE, Khoury HJ, Larson RA, Konopleva M, Cortes JE, et al: BCR-ABL1 compound mutations combining key kinase domain positions confer clinical resistance to ponatinib in $\mathrm{Ph}$ chromosome-positive leukemia. Cancer Cell 26: 428-442, 2014.

7. Shah NP, Nicoll JM, Nagar B, Gorre ME, Paquette RL, Kuriyan J and Sawyers CL: Multiple BCR-ABL kinase domain mutations confer polyclonal resistance to the tyrosine kinase inhibitor imatinib (STI571) in chronic phase and blast crisis chronic myeloid leukemia. Cancer Cell 2: 117-125, 2002.

8. Apperley JF: Part I: Mechanisms of resistance to imatinib in chronic myeloid leukaemia. Lancet Oncol 8: 1018-1029, 2007.

9. Talpaz M, Shah NP, Kantarjian H, Donato N, Nicoll J, Paquette R, Cortes J, O'Brien S, Nicaise C, Bleickardt E, et al: Dasatinib in imatinib-resistant Philadelphia chromosome-positive leukemias. N Engl J Med 354: 2531-2541, 2006.

10. O'Hare T, Shakespeare WC, Zhu X, Eide CA, Rivera VM, Wang F, Adrian LT, Zhou T, Huang WS, Xu Q, et al: AP24534, a pan-BCR-ABL inhibitor for chronic myeloid leukemia, potently inhibits the T315I mutant and overcomes mutation-based resistance. Cancer Cell 16: 401-412, 2009.

11. Poch Martell M, Sibai H, Deotare U and Lipton JH: Ponatinib in the therapy of chronic myeloid leukemia. Expert Rev Hematol 9 : 923-932, 2016.

12. Talpaz M, Kantarjian H, Kurzrock R, Trujillo JM and Gutterman JU: Interferon-alpha produces sustained cytogenetic responses in chronic myelogenous leukemia. Philadelphia chromosome-positive patients. Ann Intern Med 114: 532-538, 1991.

13. El Eit R, Itani AR, Nassar F, Rasbieh N, Jabbour M, Santina A, Zaatari G, Mahon FX, Bazarbachi A and Nasr R: Antitumor efficacy of arsenic/interferon in preclinical models of chronic myeloid leukemia resistant to tyrosine kinase inhibitors. Cancer 125: 2818-2828, 2019.

14. Zhou X, Michal JJ, Zhang L, Ding B, Lunney JK, Liu B and Jiang Z: Interferon induced IFIT family genes in host antiviral defense. Int J Biol Sci 9: 200-208, 2013.

15. Stawowczyk M, Van Scoy S, Kumar KP and Reich NC: The interferon stimulated gene 54 promotes apoptosis. J Biol Chem 286 7257-7266, 2011

16. Chen L, Liu S, Xu F, Kong Y, Wan L, Zhang Y and Zhang Z: Inhibition of proteasome activity induces aggregation of IFIT2 in the centrosome and enhances IFIT2-induced cell apoptosis Int J Biol Sci 13: 383-390, 2017.

17. Vardiman JW, Harris NL and Brunning RD: The world health organization (WHO) classification of the myeloid neoplasms Blood 100: 2292-2302, 2002.
18. Liu S, Wan J, Kong Y, Zhang Y, Wan L and Zhang Z: Inhibition of CRL-NEDD8 pathway as a new approach to enhance ATRA-induced differentiation of acute promyelocytic leukemia cells. Int J Med Sci 15: 674-681, 2018.

19. Livak KJ and Schmittgen TD: Analysis of relative gene expression data using real-time quantitative PCR and the 2(-Delta Delta $\mathrm{C}(\mathrm{T})$ ) method. Methods 25: 402-408, 2001

20. Tomoda K, Kato JY, Tatsumi E, Takahashi T, Matsuo Y and Yoneda-Kato N: The Jab1/COP9 signalosome subcomplex is a downstream mediator of Bcr-Abl kinase activity and facilitates cell-cycle progression. Blood 105: 775-783, 2005.

21. Morimoto M, Nishida T, Honda R and Yasuda H: Modification of cullin-1 by ubiquitin-like protein Nedd8 enhances the activity of SCF(skp2) toward p27(kip1). Biochem Biophys Res Commun 270: 1093-1096, 2000.

22. Chen L, Zhai W, Zheng X, Xie Q, Zhou Q, Tao M, Zhu Y, Wu C and Jiang J: Decreased IFIT2 expression promotes gastric cancer progression and predicts poor prognosis of the patients. Cell Physiol Biochem 45: 15-25, 2018.

23. Shen H, Zhan M, Zhang Y, Huang S, Xu S, Huang X, He M, Yao Y, Man M and Wang J: PLZF inhibits proliferation and metastasis of gallbladder cancer by regulating IFIT2. Cell Death Dis 9: 71, 2018

24. Ohsugi T, Yamaguchi K, Zhu C, Ikenoue T and Furukawa Y: Decreased expression of interferon-induced protein 2 (IFIT2) by $\mathrm{Wnt} / \beta$-catenin signaling confers anti-apoptotic properties to colorectal cancer cells. Oncotarget 8: 100176-100186, 2017.

25. Zhang Y, Kong Y, Liu S, Zeng L, Wan L and Zhang Z: Curcumin induces apoptosis in human leukemic cell lines through an IFIT2-dependent pathway. Cancer Biol Ther 18: 43-50, 2017.

26. Huang R, Liu H, Chen Y, He Y, Kang Q, Tu S, He Y, Zhou X, Wang L, Yang J, et al: EPS8 regulates proliferation, apoptosis and chemosensitivity in BCR-ABL positive cells via the BCR-ABL/PI3K/AKT/mTOR pathway. Oncol Rep 39: 119-128, 2018.

27. Toyoshima $\mathrm{H}$ and Hunter T: p27, a novel inhibitor of $\mathrm{G}$ cyclin-Cdk protein kinase activity, is related to p21. Cell 78: 67-74, 1994.

28. Zhang H, Peng C, Hu Y, Li H, Sheng Z, Chen Y, Sullivan C, Cerny J, Hutchinson L, Higgins A, et al: The Blk pathway functions as a tumor suppressor in chronic myeloid leukemia stem cells. Nat Genet 44: 861-871, 2012.

29. Nakao T, Geddis AE, Fox NE and Kaushansky K: PI3K/Akt/ FOXO3a pathway contributes to thrombopoietin-induced proliferation of primary megakaryocytes in vitro and in vivo via modulation of p27(Kip1). Cell Cycle 7: 257-266, 2008.

30. Shin I, Yakes FM, Rojo F, Shin NY, Bakin AV, Baselga J and Arteaga CL: PKB/Akt mediates cell-cycle progression by phosphorylation of p27(Kip1) at threonine 157 and modulation of its cellular localization. Nat Med 8: 1145-1152, 2002.

31. Wisdom R, Johnson RS and Moore C: c-Jun regulates cell cycle progression and apoptosis by distinct mechanisms. EMBO J 18: 188-197, 1999.

32. Khattar E and Kumar V: Mitogenic regulation of p27(Kip1) gene is mediated by AP-1 transcription factors. J Biol Chem 285: 4554-4561, 2010. 